\title{
Capacity Enhancement Using MIMO Antenna Arrays in Realistic Macro-Cellular Urban Environment
}

\author{
Pedro Vieira • Paula Queluz • António Rodrigues
}

Published online: 4 August 2009

C) Springer Science+Business Media, LLC. 2009

\begin{abstract}
In MIMO systems the antenna array configuration in the BS and MS has a large influence on the available channel capacity. In this paper, we first introduce a new Frequency Selective (FS) MIMO framework for macro-cells in a realistic urban environment. The MIMO channel is built over a previously developed directional channel model, which considers the terrain and clutter information in the cluster, line-of-sight and link loss calculations. Next, MIMO configuration characteristics are investigated in order to maximize capacity, mainly the number of antennas, inter-antenna spacing and SNR impact. Channel and capacity simulation results are presented for the city of Lisbon, Portugal, using different antenna configurations. Two power allocations schemes are considered, uniform distribution and FS spatial water-filling. The results suggest optimized MIMO configurations, considering the antenna array size limitations, specially at the MS side.
\end{abstract}

Keywords MIMO $\cdot$ Channel model $\cdot$ Antenna arrays $\cdot$ Water-filling

\section{Introduction}

In recent years, a lot of attention has been drawn to antenna systems with multiple elements at the Transmitter (TX) and Receiver (RX), called Multiple Input Multiple Output (MIMO)

Corresponding author e-mail address is available for all problems and questions.

P. Vieira $(\bowtie)$

Department of Electrical Engineering, Lisbon Polytechnic Institute (ISEL),

R. Conselheiro Emídio Navarro, 1, 1959-007 Lisbon, Portugal

e-mail: pvieira@deetc.isel.ipl.pt

P. Vieira $\cdot$ P. Queluz · A. Rodrigues

Instituto de Telecomunicações (IT)/IST, Technical University of Lisbon, Lisbon, Portugal

P. Queluz

e-mail: paula.queluz@1x.it.pt

A. Rodrigues

e-mail: antonio.rodrigues@1x.it.pt 
systems. The main reason is that they can achieve high spectral efficiencies [1,2]. This is particularly significant for wireless systems that are power and bandwidth limited.

The capacity advantage of MIMO channels lays in the decomposition of the channel into several spatial sub-channels, each one with different gain. The offered capacity depends directly on the sub-channel power distribution, MIMO configuration (antenna array characteristics), Signal-to-Noise-Ratio (SNR), and of course, the radio channel.

If the radio channel is unknown at the TX, uniform power allocation between the antenna elements is optimal in the capacity sense, and significant capacity gain is achieved compared with Single Input Single Output (SISO) systems. However, if the channel is known at both the RX and the TX, the channel capacity can be further increased by allocating the power in a more efficient manner, the optimum power allocation following the Shannon's principle of water-filling [3].

In this paper we propose to apply the water-filling concept in a Frequency Selective (FS) MIMO framework for macro-cells, in a realistic urban environment. The MIMO channel is built over a previously developed Directional Channel Model (DCM), which considers the terrain and clutter information in the cluster and link loss calculations [4].

The MIMO configuration characteristics are studied in order to maximize capacity, mainly the number of antennas, inter-antenna spacing and SNR dependency. The analysis is performed by investigating the distribution of the spatial/frequency eigenvalues, TX power and spectral efficiency (capacity) distributions. Uniform power allocation and spatial water-filling are implemented and compared.

Guidelines are suggested to the better MIMO configurations, in order to assure the integration of the Base Station (BS) and Mobile Station (MS) antenna systems on limited size areas. Even if the major size limitations are on the MS side, when trying to put the antennas in a small console, the real implementation of macro-cell BS antenna systems still suffers size constraints. Most of the pressure comes from the infrastructure owners where the BSs are installed, which most of the times disregard engineering recommendations in favor of aesthetics.

The paper is organized as follows. A description of antenna system, Radio Environment (RE) and mobility dependent MIMO channel model is given in Sect. 2, followed by the FS MIMO eigen and capacity review in Sects. 3 and 4, respectively. Section 5 presents the model implementation over the Lisbon urban environment. The impact of MIMO configuration and MIMO SNR is evaluated in Sects. 6 and 7, respectively. Finally, the conclusions are presented in Sect. 8.

\section{The MIMO Channel Model}

The MIMO concept is defined, throughout this paper, as a radio link with $R$ antennas at the BS and $S$ antennas at the MS, as pictured in Fig. 1. For the downlink, the TX and RX antennas are at the BS and MS respectively, while for uplink, the roles are reversed.

The received signal vector $\mathbf{y}(f)$ at the MS antenna array is denoted by,

$$
\mathbf{y}(f)=\left[y_{1}(f), y_{2}(f), \ldots, y_{S}(f)\right]^{T}
$$

where $y_{s}(f)$ is the signal at the $s$ th antenna element, $[\ldots]^{T}$ denotes the transpose operation and $f$ is the carrier frequency. Similarly, the transmitted signals at the BS, $x_{r}(f)$, define the vector $\mathbf{x}(f)$,

$$
\mathbf{x}(f)=\left[x_{1}(f), x_{2}(f), \ldots, x_{R}(f)\right]^{T} .
$$




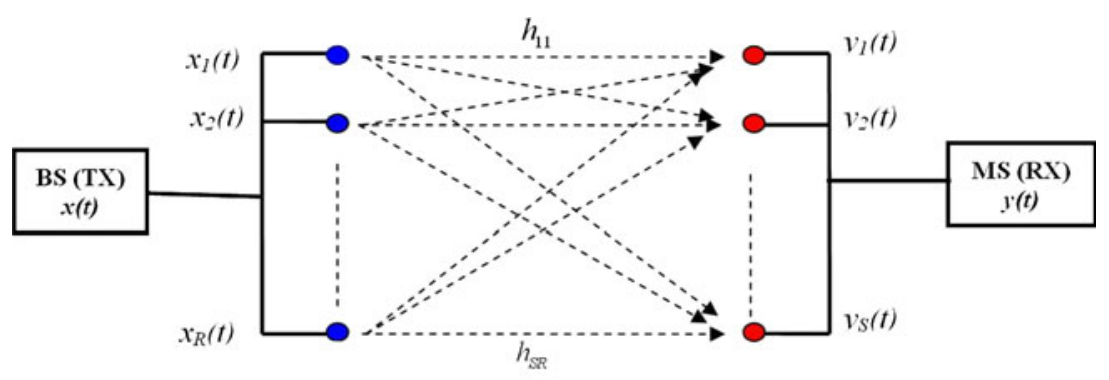

$R$ elements

Propagation Channel

$S$ elements

Fig. 1 The MIMO setup

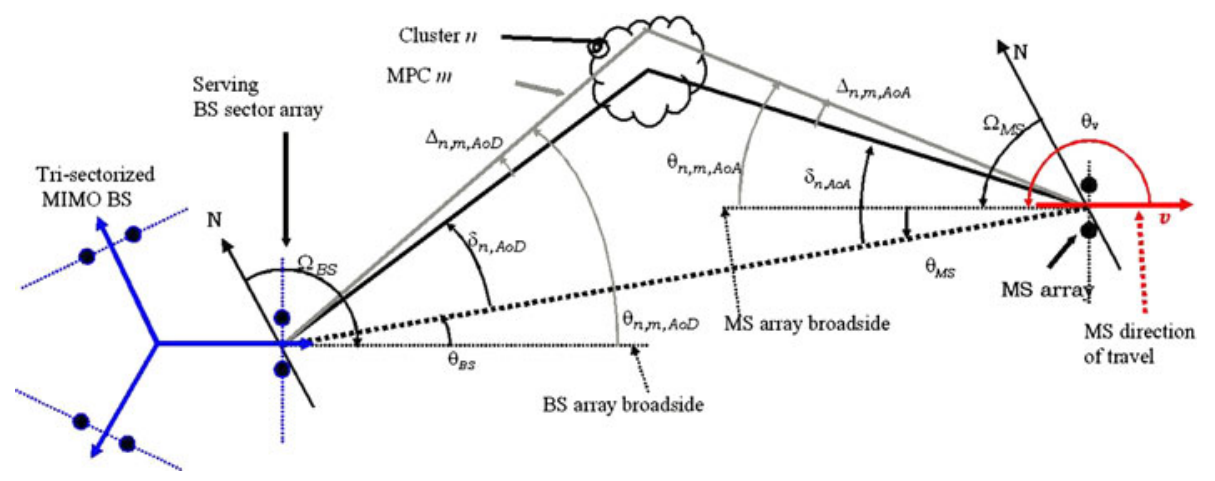

Fig. 2 BS and MS MIMO angle parameters

The vectors $\mathbf{y}(f)$ and $\mathbf{x}(f)$ are linked by the following expression,

$$
\mathbf{y}(f)=\mathbf{H}(f) \mathbf{x}(f)+\mathbf{n}(f)
$$

where $\mathbf{n}(f)$ is additive white Gaussian noise and $\mathbf{H}(f) \in \mathbf{C}^{S \times R}$ is the instantaneous MIMO radio channel matrix, i.e., refers to a radio propagation channel snapshot. $\mathbf{H}(f)$ describes the connections between the BS and the MS and depends directly on the radio propagation environment.

The RE characterization will be set by the extended European COoperation in the field of Scientific and Technical research (COST) 273 DCM [5,6]. According to the model, the received signal at the MS consists of several time-delayed multi-path replicas of the transmitted signal which are grouped in clusters. The first cluster is always around the MS. The $N$ clusters are defined by power, delay, Angle of Departure (AoD), Angle of Arrival (AoA) and are determined stochastically according to the channel model generation procedure. Each cluster consists of $M$ Multi-Path Component (MPC)s. Figure 2 shows the angular parameters used in the model. The BS is assumed to be multi-sectored. Each sector is equipped with a multi element array antenna which, along with the MS array, will build up the MIMO setup.

The MS travels along several BSs service area. The MS positional data is generated using a new mobility model developed and validated [7] for Lisbon city. After each MS movement, all the MS parameterization is stored, mainly time reference, latitude/longitude positioning, speed and direction of travel. 
In a certain time instant, a BS sector will be the serving one, according to a classic signal strength based locating algorithm.

The following definitions are used, as in [8]:

- $\Omega_{B S}$, BS antenna array orientation, defined as the difference between the broadside of the BS array and the absolute North $(\mathrm{N})$ reference direction;

- $\theta_{B S}$, Line of Sight (LoS) AoD direction between the BS and MS, with respect to the broadside of the BS array;

- $\delta_{n, \mathrm{AoD}}$, AoD for the $n$th $(n=1 \ldots N)$ cluster with respect to the $\operatorname{LoS} \operatorname{AoD} \theta_{0}$;

- $\Delta_{n, m, \mathrm{AoD}}$, Offset for the $m$ th $(m=1 \ldots M)$ MPC of the $n$th cluster with respect to $\delta_{n, \mathrm{AoD}}$

- $\theta_{n, m, \text { AoD }}$, Absolute AoD for the $m$ th $(m=1 \ldots M)$ MPC of the $n$th cluster at the BS with respect to the BS broadside;

- $\Omega_{M S}$, MS antenna array orientation, defined as the difference between the broadside of the MS array and the absolute North reference direction;

- $\theta_{M S}$, Angle between the BS-MS LoS and the MS broadside;

- $\delta_{n, \text { AoA }}$, AoA for the $n$th $(n=1 \ldots N)$ cluster with respect to the $\operatorname{LoS}$ AoA $\theta_{0, M S}$;

- $\Delta_{n, m, \mathrm{AoA}}$, Offset for the $m$ th $(m=1 \ldots M)$ MPC of the $n$th cluster with respect to $\delta_{n, \mathrm{AoA}}$

- $\theta_{n, m, \text { AoA }}$ Absolute AoA for the $m$ th $(m=1 \ldots M)$ MPC of the $n$th cluster at the MS with respect to the MS broadside;

- $\vec{v}$, MS speed vector, given by the MS mobility model;

- $\theta_{v}$, Angle of the speed vector with respect to the MS broadside $\theta_{v}=\arg (\vec{v})$.

In order to calculate the MIMO channel matrix $\mathbf{H}$, an evaluation technique that requires only a single measurement of the channel is proposed [9]. This technique relies on the fact that different realizations of the transfer function can be generated by changing the phases of the MPCs. It is a well-established fact in mobile radio that these phases are stochastically distributed random variables, whose different realizations occur as either TX, RX, or scatterers move [10].

The proposed MIMO channel model is presented. For an $R$ element linear BS array and a $S$ element linear MS array, the channel coefficients for $N$ clusters each one of them with $M$ MPCs are given by a $S \times R$ matrix of complex amplitudes. The $(s, r)$ th component $(s=1, \ldots, S ; r=1, \ldots, R)$ of $\mathbf{H}$ is calculated generating different realizations of the transfer function from the $r$ th TX antenna to the $s$ th RX antenna as

$$
\begin{aligned}
h_{s, r}(f)= & \sum_{l=1}^{L} \sum_{n=1}^{N} \sum_{m=1}^{M} a_{n, m} \sqrt{G_{B S, r}\left(\theta_{n, m, \mathrm{AoD}}\right)} \\
& \times \exp \left(-j k d_{r} \sin \left(\theta_{n, m, \mathrm{AoD}}\right)\right) \sqrt{G_{M S, s}\left(\theta_{n, m, \mathrm{AoA}}\right)} \\
& \times \exp \left(-j k d_{s} \sin \left(\theta_{n, m, \mathrm{AoA}}\right)\right) \exp \left(-j 2 \pi f \tau_{l}\right) \\
& \times \exp \left(-j k\|\overrightarrow{\mathbf{v}}\| \cos \left(\theta_{n, m, \mathrm{AoA}}-\theta_{v}\right)\right) \times g\left(\tau_{n, m}, \tau_{l}\right)
\end{aligned}
$$

where

- $L$ is the number of delay taps;

- $\quad N$ and $M$ are the number of clusters and MPCs per cluster, respectively;

- $G_{B S, r}\left(\theta_{n, m, \mathrm{AoD}}\right)$ is the $r$ th BS antenna gain pattern of each array element;

- $G_{M S, s}\left(\theta_{n, m, \mathrm{AoA}}\right)$ is the $s$ th MS antenna gain pattern of each array element;

- $a_{n, m}$ is the complex amplitude of the $(n, m)$ th MPC; 
$-\quad j$ is the square root of -1 ;

- $k$ is the wave number $2 \pi / \lambda$ where $\lambda$ is the carrier wavelength in meters; $d_{r}$ is the distance in meters from BS antenna element $r$ to the reference antenna (for the reference antenna $\left.r=1, d_{1}=0\right) ; d_{s}$ is the distance in meters from MS antenna element $s$ to the reference antenna (for the reference antenna $s=1, d_{1}=0$ ); $\|\overrightarrow{\mathbf{v}}\|$ is the magnitude of the MS speed vector; $\theta_{v}$ is the angle of the MS speed vector;

$g\left(\tau_{n, m}, \tau_{l}\right)$ is an auxiliary function given by

$$
g\left(\tau_{n, m}, \tau_{l}\right)= \begin{cases}1 & \tau_{n, m} \in\left[\tau_{l-1}, \tau_{l}\right] \\ 0 & \text { other }\end{cases}
$$

where $\tau_{n, m}$ is the $(n, m)$ th MPC excess delay referred to the LoS radio path and $\tau_{l}$ is the $l$ th tap delay which is entirely dependent on the system bandwidth, $B$, as

$$
\tau_{l}=\frac{1}{B} \text {. }
$$

Using this approach the MIMO channel model implementation will depend on the bandwidth and resolvable MPCs. It is well known that the system bandwidth affects small-scale fading parameters, which influences MIMO performance [9]. Thus, the bandwidth effect must be added.

No real antenna radiation patterns were used. Due to the model complexity and the simulation time associated to the radiation pattern interpolation calculations, 3rd Generation Partnership Project (3GPP) simplified antenna model was used [8].

The proposed MIMO channel model is therefore, system, radio environment, mobility and frequency dependent. The MIMO channel will depend on the system configuration: number of antennas, antenna spacing, antenna radiation pattern and system bandwidth. The radio environment realization is introduced using a realistic macro-cell directional model [11]. Additionally, the MS mobility [7] guarantees a realistic navigation through the BS service area. The Doppler shift is introduced using the MS speed vector information.

We can, thus, generate different realizations of the channel matrix $\mathbf{H}$,

$$
\mathbf{H}(f)=\left[\begin{array}{llll}
h_{11}(f) & h_{12}(f) & \ldots & h_{1 R}(f) \\
h_{21}(f) & \ldots & & \ldots \\
\ldots & & \ldots & \ldots \\
\ldots & & & \ldots \\
h_{S 1}(f) & \ldots & \ldots & h_{S R}(f)
\end{array}\right] \in C^{S \times R}
$$

using the following steps:

1. From a single channel realization, i.e., a single snapshot of the channel matrix, determine the cluster/MPCs delays, AoDs, AoAs and amplitude [4];

2. Extract the MS positioning information for that particular location (speed vector);

3. Compute synthetically the impulse responses at the positions of the antenna elements, and at different frequencies.

For each channel realization, the capacity (in bit/s/Hz) can be computed as [1],

$$
C=\log _{2} \operatorname{det}\left(\mathbf{I}+\frac{\rho}{S} \mathbf{H}^{H} \mathbf{H}\right)
$$

where $\rho$ denotes the SNR. I is the identity matrix and superscript $H$ means Hermitian transposition. For the FS case, the capacity has to be evaluated by integrating over all frequencies. In the following, the channel capacity per unit bandwidth (in bit/s/ Hz) is considered: 


$$
C=\frac{1}{B} \int_{B} \log _{2} \operatorname{det}\left(\mathbf{I}+\frac{\rho}{S} \mathbf{H}^{H}(f) \mathbf{H}(f)\right) \mathrm{d} f .
$$

Here, $\mathbf{H}(f)$ is the frequency-dependent transfer matrix and the integration range is the system bandwidth $B$.

\section{The MIMO Eigen-Analysis}

One of the motivations for implementing MIMO technology in wireless systems is the possibility to achieve orthogonal sub-channels between the two ends of the path in a scattering environment and subsequently to increase the offered capacity. The concept of orthogonality emphasizes the strength of the MIMO system since it indicates that these multiple channels are independent from each other.

Considering a frequency selective channel and in order to proceed with an adequate eigen-analysis treatment, the channel bandwidth is divided into a number of flat-fading subchannels $\mathbf{H}\left(f_{q}\right)$, where $f_{q}$ is the $q$ th sub-carrier central frequency. Let $Q$ be the total number of sub-carriers.

The number of sub-channels between two terminals can be computed by using Singular Value Decomposition (SVD) of matrix $\mathbf{H}\left(f_{q}\right)$ or the Eigenvalue Decomposition (EVD) [12] of the instantaneous correlation matrix $\mathbf{R}\left(f_{q}\right)$ defined as,

$$
\mathbf{R}\left(f_{q}\right)=\left\{\begin{array}{cc}
\mathbf{H}\left(f_{q}\right) \mathbf{H}\left(f_{q}\right)^{H}, & S \leq R \\
\mathbf{H}\left(f_{q}\right)^{H} \mathbf{H}\left(f_{q}\right), & R<S \\
\cdot &
\end{array}\right.
$$

The calculation of the parallel independent channels is summarized below, where $\mathbf{U}\left(f_{q}\right)$ and $\mathbf{V}\left(f_{q}\right)$ are unitary matrices, $\Sigma\left(f_{q}\right)$ and $\Gamma\left(f_{q}\right)$ diagonal matrices and $\mathbf{u}\left(f_{q}\right), \mathbf{v}\left(f_{q}\right)$ are the left and right singular vectors, respectively.

An important relationship between the SVD of $\mathbf{H}\left(f_{q}\right)$ and the EVD of $\mathbf{R}\left(f_{q}\right)$ is that $\gamma_{k, q}=\sigma_{k, q}^{2}$ where $\sigma_{k, q}$ and $\gamma_{k, q}$ are the $k$ th singular value and eigenvalue of $\mathbf{H}\left(f_{q}\right)$ and $\mathbf{R}\left(f_{q}\right)$, respectively. $\Gamma_{i j}$ denotes the elements of matrix $\Gamma$.

\section{SVD (Singular Value Decomposition)}

$$
\mathbf{H}\left(f_{q}\right)=\mathbf{U}\left(f_{q}\right) \Sigma\left(f_{q}\right) \mathbf{V}\left(f_{q}\right)^{H}
$$

where

$$
\begin{gathered}
\Sigma\left(f_{q}\right)=\text { diagonal }\left(\sigma_{1, q}, \sigma_{2, q}, \ldots, \sigma_{k, q}\right) \\
\sigma_{1, q} \geq \sigma_{2, q} \geq \cdots \geq \sigma_{k, q} \geq 0
\end{gathered}
$$

\section{EDV (Eigenvalue Decomposition)}

$$
\begin{aligned}
\mathbf{H}\left(f_{q}\right) \mathbf{H}\left(f_{q}\right)^{H} & =\mathbf{U}\left(f_{q}\right) \Gamma\left(f_{q}\right) \mathbf{U}\left(f_{q}\right)^{H} \\
\mathbf{H}\left(f_{q}\right)^{H} \mathbf{H}\left(f_{q}\right) & =\mathbf{V}\left(f_{q}\right) \Gamma\left(f_{q}\right) \mathbf{V}\left(f_{q}\right)^{H} \\
\Gamma_{i j}\left(f_{q}\right) & =\left(\Sigma_{i j}\left(f_{q}\right)\right)^{2}
\end{aligned}
$$




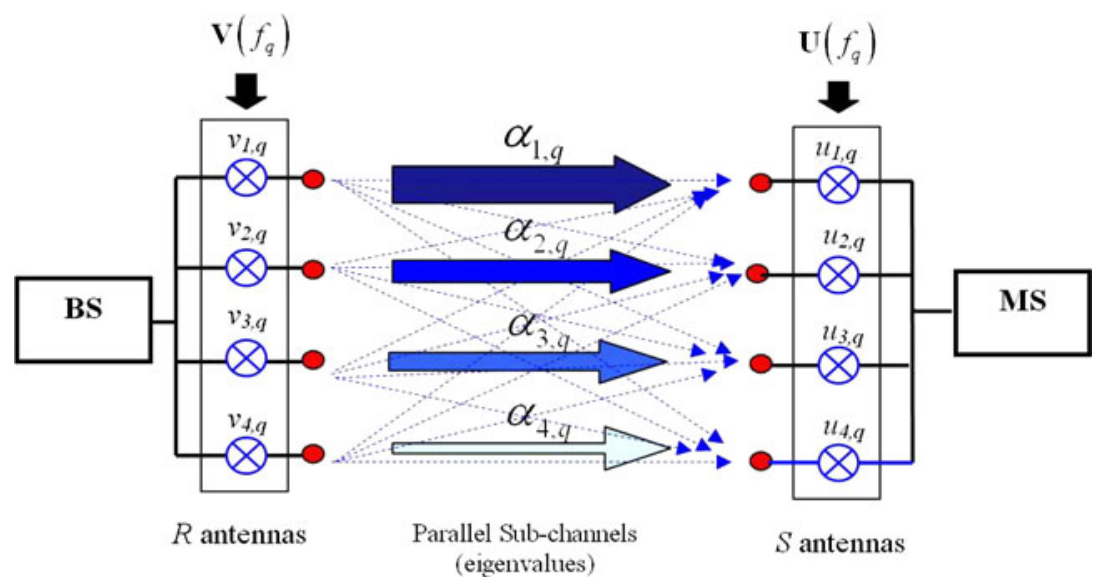

Fig. 3 Example of a $4 \times 4$ MIMO antenna topology. Parallel sub-channels (eigenvalues)

where

$$
\begin{aligned}
& \Gamma\left(f_{q}\right)=\operatorname{diagonal}\left(\gamma_{1, q}, \gamma_{2, q}, \ldots, \gamma_{k, q}\right) \\
& \gamma_{1, q} \geq \gamma_{2, q} \geq \cdots \geq \gamma_{k, q} \geq 0
\end{aligned}
$$

with

$$
\begin{aligned}
& \mathbf{U}\left(f_{q}\right)=\left[\mathbf{u}_{1, q}, \ldots, \mathbf{u}_{S, q}\right] \in C^{S \times S} \\
& \mathbf{V}\left(f_{q}\right)=\left[\mathbf{v}_{1, q}, \ldots, \mathbf{v}_{R, q}\right] \in C^{R \times R}
\end{aligned}
$$

The SVD is an appropriate way of diagonalising matrix $\mathbf{H}\left(f_{q}\right)$ and finding the eigenvalues [12]. The $k$ th eigenvalue can be interpreted as the power gain of the $k$ th sub-channel. In the following analysis, the eigenvalues are normalized to the mean power of a single TX and RX channel, $\Lambda_{q}$, equivalent to the frequency domain definition,

$$
\begin{gathered}
\Lambda_{q}=\frac{1}{S R} \sum_{s=1}^{S} \sum_{r=1}^{R}\left|h_{s r}\left(f_{q}\right)\right|^{2} \\
\alpha_{k, q}=\frac{\gamma_{k, q}}{\Lambda_{q}}
\end{gathered}
$$

Irrespective of the numerical method used to perform the analysis, a channel matrix $\mathbf{H}\left(f_{q}\right)$ may offer $K_{q}$ parallel sub-channels with different power gains, $\alpha_{k, q}$, where

$$
K_{q}=\operatorname{Rank}\left(\mathbf{R}\left(f_{q}\right)\right) \leq \operatorname{Min}(S, R)
$$

and the functions $\operatorname{Rank}()$ and $\operatorname{Min}()$ return the rank of the matrix and the minimum value of the arguments, respectively.

Figure 3 illustrates a $4 \times 4 \mathrm{MIMO}$ antenna topology. In such a configuration, and for each sub-carrier $f_{q}, 16$ radio links are created, but only 4 orthogonal sub-channels with power gain $\alpha_{k, q}(k=1, \ldots, 4)$, are defined. The difference in gain between the parallel sub-channels is symbolized by the thickness of the presented arrows.

In engineering terms, $\mathbf{u}_{\mathbf{s}, \mathbf{q}}$ and $\mathbf{v}_{\mathbf{r}, \mathbf{q}}$ are referred to as the weight vectors. To get the weight vectors, it is numerically more convenient to use the SVD. Based on this operation, eigenvalues will be calculated in the following. However, if this technique is to be optimal on a 
real system implementation, the $\mathbf{u}_{\mathbf{s}, \mathbf{q}}$ and $\mathbf{v}_{\mathbf{r}, \mathbf{q}}$ values have to be applied to the respective ends of the link. Consequently, the eigenvalue method is only useful when the channel is known, i.e., when the information of the radio channels is available at both TX and RX.

\section{MIMO Channel Capacity}

The concept of capacity in FS MIMO channels is investigated in this section. The presented values correspond to the theoretical maximum amount of information that can be transmitted over a bandwidth limited channel. In real systems, the achievable capacity is normally limited due to coding, detection and constellation size, among others. Therefore, the capacity results in this section have to be seen as upper bound values.

Following the previous eigen-analysis, the channel bandwidth is split into $Q$ flat-fading sub-channels $\mathbf{H}\left(f_{q}\right)$. In the situation where the channel is known at both TX and RX and is used to compute the antennnas optimum weight, the power gain in the $(k, q)$ th eigenmode channel is given by the $\alpha_{k, q}$ eigenvalue, i.e., the SNR for the $(k, q)$ th sub-channel, $v_{k, q}$, is equal to [13],

$$
v_{k, q}=\alpha_{k, q} \frac{p_{k, q}}{\sigma_{N}^{2}}
$$

where $p_{k, q}$ is the power assigned to the $(k, q)$ th sub-channel, and $\sigma_{N}^{2}$ is the noise power. In order to evaluate the performance of different power allocation methods, we use the normalized Shannon channel capacity (in bit/s/Hz). For the $q$ th sub-channel, it can be expressed as [3],

$$
C_{q}=\sum_{k=1}^{M} \log _{2}\left(1+v_{k, q}\right)=\sum_{k=1}^{M} \log _{2}\left(1+\alpha_{k, q} \frac{p_{k, q}}{\sigma_{N}^{2}}\right) .
$$

Hence, the total normalized MIMO channel capacity for a frequency-selective channel using the discretization of equation (9) is,

$$
C=\frac{1}{Q} \sum_{q=1}^{Q} \sum_{k=1}^{K_{q}} \log _{2}\left(1+\alpha_{k, q} \frac{p_{k, q}}{\sigma_{N}^{2}}\right) .
$$

Given the set of eigenvalues $\alpha_{1, q}, \alpha_{2, q}, \ldots, \alpha_{K_{q}, q}$ the power $p_{k, q}$ that should be allocated to each spatial sub-channel $(k, q)$ in order to maximize the capacity can be determined using Gallager's water-filling theorem. This will be named as spatial water-filling.

Other possibility toward capacity enhancement evolves water-filling in the frequency domain. Preliminary simulations allow to conclude that the gain from water-filling in the frequency domain, i.e., distributing power between the $Q$ sub-frequencies, is negligible, compared with a uniform power distribution. Additionally, the hybrid form usually named joint space-frequency water-filling does not create an added value in terms of capacity, compared with the spatial water-filling, while the implementation complexity is greater. Similar conclusions were reached in [14]. Hence, the implemented solution considers spatial waterfilling only, over a frequency selective channel. The algorithm assigns equal power to all the $Q$ sub-channels considering the power constraint,

$$
\frac{P_{T x}}{Q}=\sum_{k=1}^{K_{q}} p_{k, q}
$$


where $P_{T x}$ is the total transmitted power.

Assuming all noise powers to be the same, the "water-filling" concept is the solution to the maximum capacity, where each channel is filled up to a common level, $D_{q}$,

$$
\frac{1}{\alpha_{1, q}}+p_{1, q}=\frac{1}{\alpha_{2, q}}+p_{2, q}=\cdots=\frac{1}{\alpha_{k, q}}+p_{k, q}=D_{q} .
$$

Thus, the channel with the highest gain receives the largest share of the power. The power level calculation as a function of the eigenvalues has to be executed iteratively. Assuming the previous constraints the system can be described by the set of equations:

$$
\begin{aligned}
& p_{k, q}=D_{q}-\frac{1}{\alpha_{k, q}} \\
& \sum_{k=1}^{K_{q}} p_{k, q}=\frac{P_{T x}}{Q} .
\end{aligned}
$$

Considering that all sub-channels are active, and using (25), the water level is obtained as,

$$
D_{q}=\frac{\frac{P_{T x}}{Q}+\sum_{k} \frac{1}{\alpha_{k, q}}}{K_{q}}
$$

from which the power values cab be straightforwardly computed.

If all the powers are non-negative, the problem is solved. Otherwise, some of the $K_{q}$ subchannels have to be removed, i.e., their power has to be set to zero, and the process has to be repeated until no negative power arises. After setting the power of the $i$ th sub-channel to zero, the new "water level" has to be calculated using the expression,

$$
D_{q, \text { new }}=\frac{\frac{P_{T x}}{Q}+\sum_{k}^{K_{q}} \frac{1}{\alpha_{k, q}}-\frac{1}{\alpha_{i, q}}}{K_{q}-1} .
$$

After the previous step the algorithm must be restarted all over again, converging to the final solution where all the powers associated to the sub-channels are non negative.

The water-filling is of course dependent on the knowledge of the channels on the transmit side. In the case where the channel is unknown at the transmitter, the only reasonable division of power is a uniform distribution over the antennas and frequencies, i.e.,

$$
p_{k, q}=\frac{P_{T x}}{R \cdot Q} \text {. }
$$

\section{MIMO Channel Model Implementation}

The MIMO channel model simulator was developed in MATLAB $^{(}$and requires building data information, terrain data, street data, BS and MS information. The BS and MS data store the geographical positioning, height, power parameters, MIMO antenna configuration, radiation pattern, and system parameters like handover hysteresis and offset. The MS positional data is generated using a new mobility model developed and validated [7] for Lisbon city.

The considered DCM [11], used as an input to the MIMO frequency selective channel construction, imports cartographic data to dynamically introduce terrain and clutter effects on path-loss prediction [5] and MPC cluster generation [6]. The channel model framework was developed and validated [4] in order to increase realism in the radio channel behavior over an irregular terrain plus clutter environment. 
In the sequence, a simplified locating algorithm is implemented using hard handover, which enables to choose the MSs' best servers.

For each BS-MS radio-link, the new DCM is applied. The MS local movement is compared with the size of the local area, which is defined as the area over which all large-scale fading parameters can be viewed as constants. The large-scale parameters are updated when the MS movement exceeds the size of the local area, which was set to $20 \lambda$, being $\lambda$ the carrier wavelength.

The estimated minimum coherence time is $4.8 \mathrm{~ms}$, which gives a $3 \mathrm{~cm}$ channel coherent travel distance at $20 \mathrm{~km} / \mathrm{h}$ average speed. The speed was calculated from the mobility model. In the following, for each $20 \lambda=300 \mathrm{~cm}$ local area size (at $2 \mathrm{GHz}$ ), 100 channel realizations were generated.

The eigenvalue and capacity calculations were performed for $Q=100$ sub-channels with a $200 \mathrm{kHz}$ individual bandwidth. Hence, the total bandwidth $B$ is $20 \mathrm{MHz}$. The sub-channel bandwidth is equal to the channel coherence bandwidth considering a $1 \mu$ s calculated delay spread.

The tested urban RE is located in the city of Lisbon, Portugal, and several virtual test MSs travel around the city for a period of $10 \mathrm{~min}$. To this end, a large number of MIMO channel realizations were generated for each cluster oriented large-scale fading set of parameters, that simulate the MIMO channel conditions as the MS moves connected at the BS.

\section{A MIMO 4 × 4 Illustrative Case-Study}

In order to illustrate the distribution of the channel capacity and the gain that can be expected from spatial water-filling, we therefore start by analyzing the Probability Density Function (PDF) of the eigenvalues for the already mentioned urban FS MIMO scenario. The eigenvalue distribution is shown for an illustrative $4 \times 4$ MIMO channel, $Q=4$ subchannels with an individual bandwidth of $5 \mathrm{MHz}$ at a $\mathrm{SNR}$ of $5 \mathrm{~dB}$. Considering the total bandwidth $B=20 \mathrm{MHz}$, there are in total $K=16$ eigenvalues of frequency selective channel $\mathbf{H}$.

The $5 \mathrm{MHz}$ sub-channel bandwidth was chosen in order to contain the number of subchannels under a small value, and to enable readable graphical representation. The general simulations with $Q=100$, times the number of eigenmodes, turn graphical analysis too messy.

The Cumulative Distribution Function (CDF)s of the 16 ordered amplitudes are shown in Fig. 4. The four CDF curves for each $k$ spatial sub-channel have similar behaviors. For this reason, they seem overlapping, even though a small dispersion is noticed around the lines.

The stronger eigenvalues are $\alpha_{1, q}$ and $\alpha_{2, q}$ with approximately 11 and $3 \mathrm{~dB}$ mean values, respectively. The remaining ones, $\alpha_{3, q}$ and $\alpha_{4, q}$ are definely weaker with -4 and $-14 \mathrm{~dB}$ mean values, respectively (see Fig. 5).

The power distribution between the eigen-channels can be analyzed in Figs. 6 and 7 . For uniform power allocation all eigen-modes are assigned the same power. So, each subchannel gets $1 / 16=6.25 \%$ of the power. For the spatial water-filling, the power according to the water-filling rule is assigned to the 16 sub-channels. In this case, only 10 out of 16 sub-channels will actually be used.

The presented upper bound capacity calculations using the two power allocation strategies were added to the already existing MATLAB ${ }^{\complement}$ developed MIMO simulator and further calculations for the same macro-cell urban scenario were performed. 


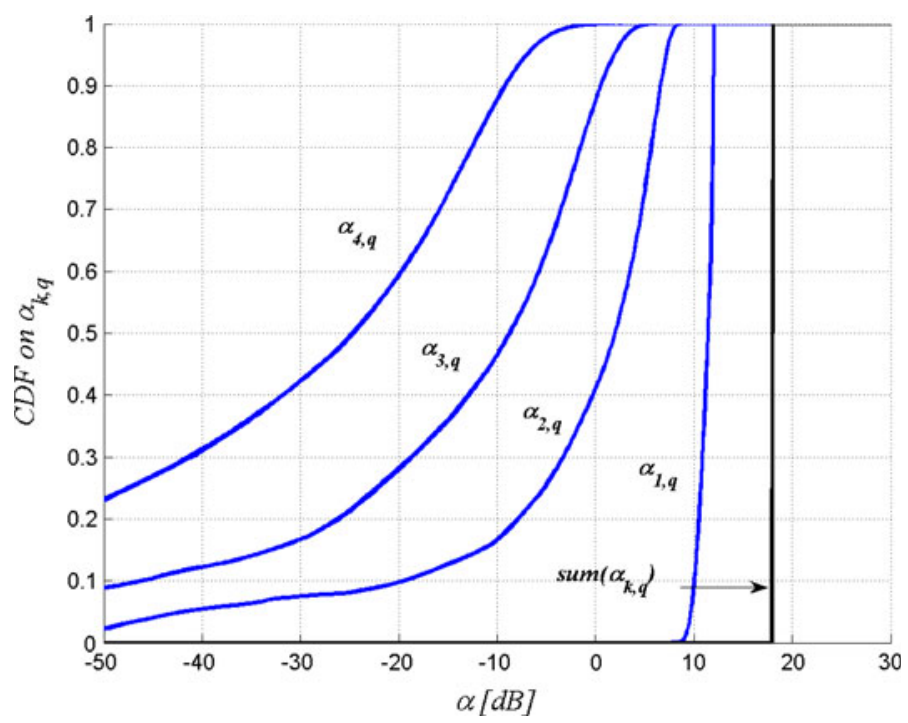

Fig. 4 Eigenvalue CDF for a $4 \times 4$ MIMO antenna topology and 4 sub-channels $\left(K_{\mathrm{q}}=4, Q=4\right)$, $\mathrm{SNR}=5 \mathrm{~dB}$

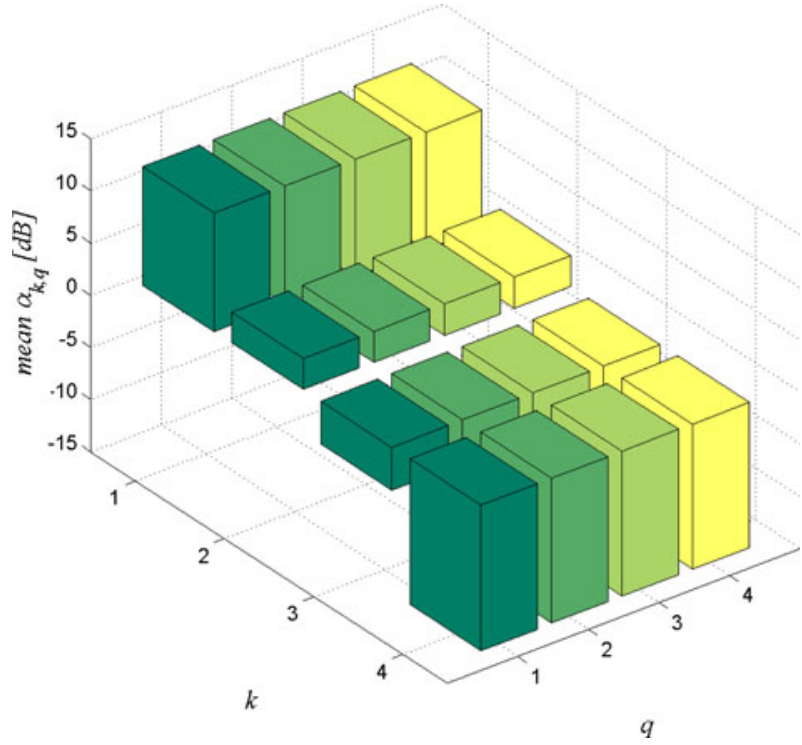

Fig. 5 Mean eigenvalues, $\alpha_{k, q}$ for a $4 \times 4$ MIMO antenna topology and 4 sub-channels $\left(K_{\mathrm{q}}=4, Q=4\right)$, $\mathrm{SNR}=5 \mathrm{~dB}$

Figure 8 illustrates the impact of each $(k, q)$ sub-channel CDF upon the total capacity available for the same $4 \times 4$ MIMO setup assuming a $5 \mathrm{~dB}$ SNR, and for the two power allocation techniques. The mean values for the individual $(k, q)$ sub-channels capacities are presented in Figs. 9 and 10. 


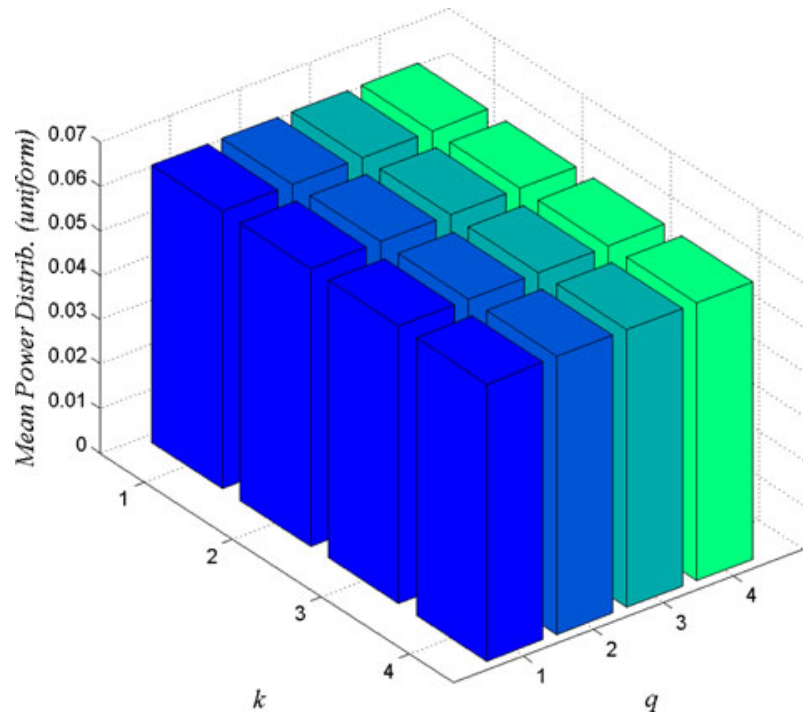

Fig. 6 Mean power, $p_{k, q}$ uniform distribution for a $4 \times 4$ MIMO antenna topology and 4 sub-channels $\left(K_{\mathrm{q}}=4, Q=4\right), \mathrm{SNR}=5 \mathrm{~dB}$

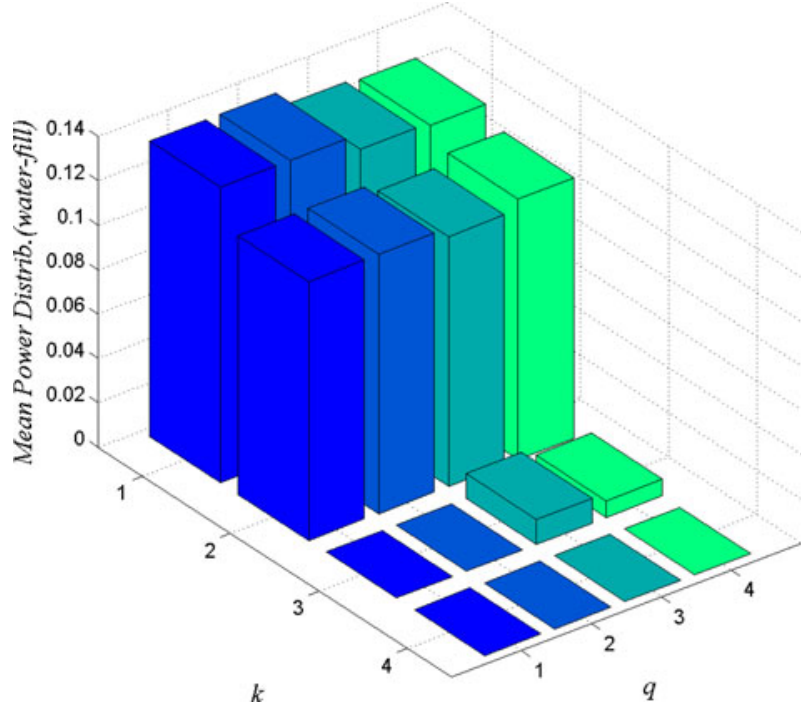

Fig. 7 Mean power, $p_{k, q}$ water-filling distribution for a $4 \times 4$ MIMO antenna topology and 4 sub-channels $\left(K_{\mathrm{q}}=4, Q=4\right), \mathrm{SNR}=5 \mathrm{~dB}$

The water-filling scheme always provides a higher capacity when compared with the uniform power distribution. When calculating the mean values, one can conclude that the the total capacity for a uniform and water-filling strategy is 5.0 and $6.1 \mathrm{bit} / \mathrm{s} / \mathrm{Hz}$, respectively, which gives a $22 \%$ considerable increase in mean capacity. Hence, spatial water-filling really improves capacity and it is a promising technique when channel information is available at the TX. 


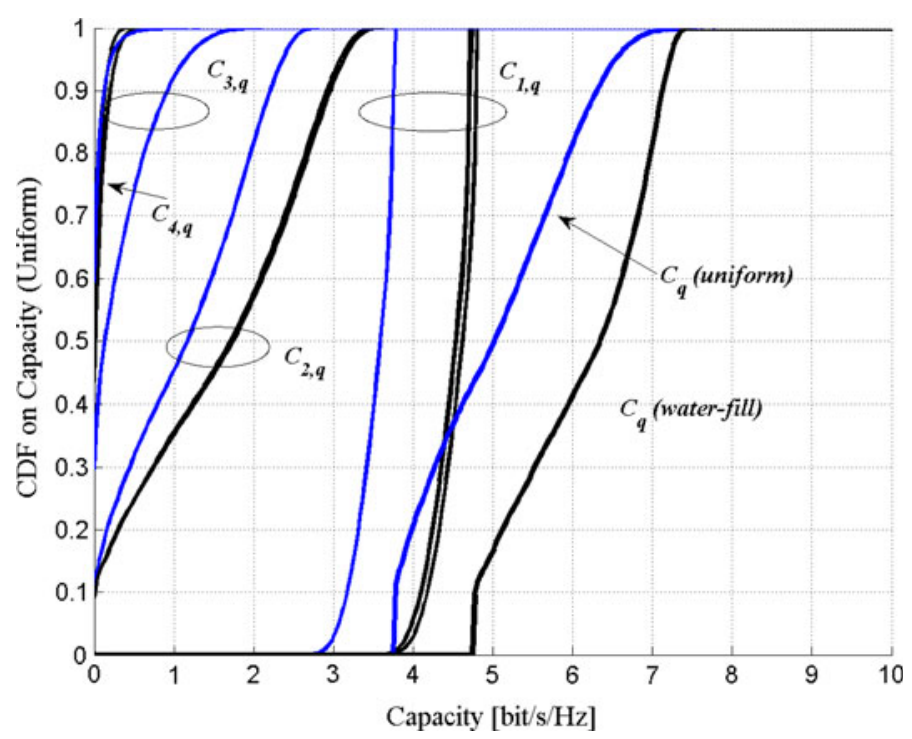

Fig. $8 \mathrm{CDF}$ of the capacity per sub-channel $C_{k, q}$ and total capacity $C_{q}$ for MIMO $4 \times 4$ using uniform (blue) and spatial water-filling (black) power allocation $(\mathrm{SNR}=5 \mathrm{~dB}$ )

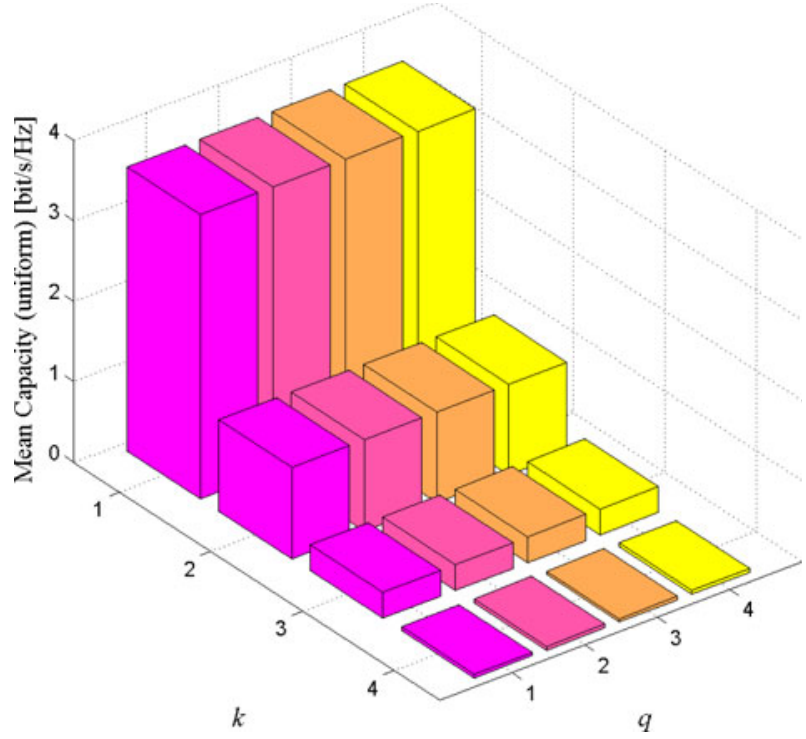

Fig. 9 Mean capacity per sub-channel $C_{k, q}$ for MIMO $4 \times 4$ and uniform power allocation ( $\mathrm{SNR}=5 \mathrm{~dB}$ )

\section{MIMO Configuration Impact}

The current section presents the MIMO configuration impact on channel capacity. The considered MIMO parameters are the number of antennas and BS/MS antenna spacing. The simulated MIMO configurations will be named in the following figures using a label, which is, using previous terminology, $R\left(d_{r}\right) \times S\left(d_{s}\right)$. 


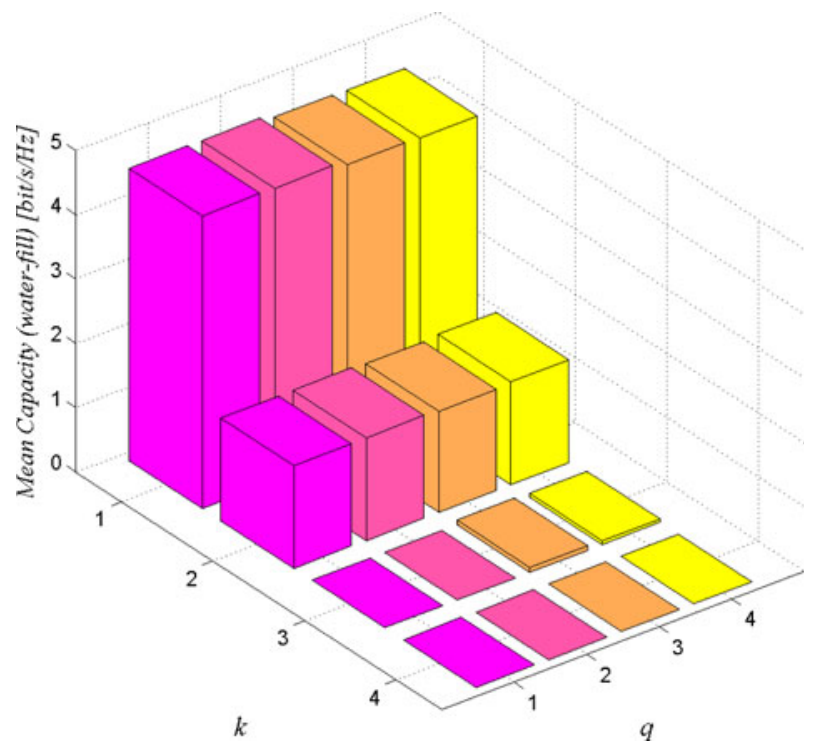

Fig. 10 Mean capacity per sub-channel $C_{k, q}$ for MIMO $4 \times 4$ and water-filling power allocation $(\mathrm{SNR}=5$ $\mathrm{dB})$

The conclusions and recommendations are supported by eigen analysis and capacity results using spatial water-filling and uniform power allocation.

\subsection{Number of Antenna Elements Impact}

Several half wavelength ( $0.5 \lambda)$ spaced MIMO configurations were chosen and compared with the reference SISO. The BS and MS number of antenna elements is equal and varies from one to seven. The MIMO gain is defined as the relation between the MIMO configuration mean capacity and the SISO mean capacity. Figure 11 presents the MIMO gain using the two implemented power allocation techniques. The water-fill gain relates, for each MIMO configuration, the mean capacity using spatial water-fill with the equivalent mean capacity using a uniform power allocation.

The MIMO gain increases with the number of antenna elements. Starting with an already outstanding $80 \%$ increase using a simple $2 \times 2$ MIMO configuration, the mean capacity gain can reach $380 \%$ using MIMO $7 \times 7$ with spatial water-filling.

The capacity gain from spatial water-filling compared to the capacity from uniform power allocation is considerable (a $30 \%$ increase was noticed), for all the configurations.

The waste of power when using uniform power allocation is more severe when really poor eigen-mode channels are evident, like the presented macro-cell case, where the second eigenchannel can be $20 \mathrm{~dB}$ smaller than the first one. In fact, the capacity gain from water-filling increases with the difference between the eigenvalues.

\subsection{BS Antenna Spacing}

For the BS antenna element spacing study, the MS antenna spacing is kept at half of the wavelength $(0.5 \lambda)$. The 3 GPP [8] suggests several BS antenna spacings from 0.5 to $10 \lambda$. 


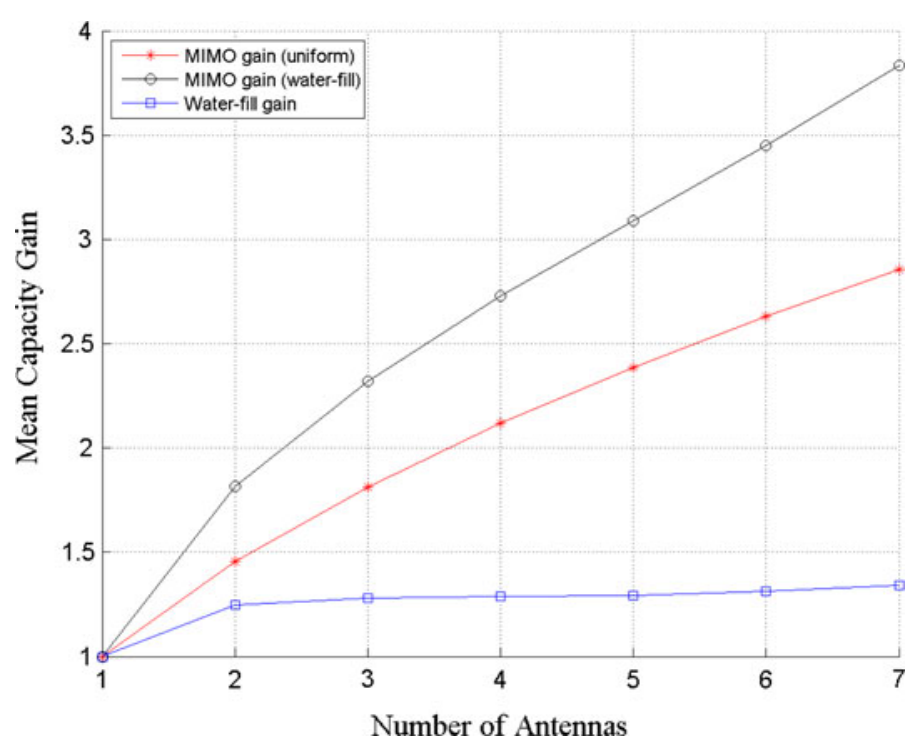

Fig. 11 MIMO and water-fill gain for MIMO $m \times m$ setup, $m=1, \ldots, 7$. SNR $=5 \mathrm{~dB}$, half of wavelength antenna spacing

Hence, the proposed test is to vary the BS antenna spacing from $0.25 \lambda$ to near $10 \lambda$ analyzing several MIMO configurations.

In terms of antenna elements number, the MIMO $3 \times 3$ setup is presented. Other configurations were tested and the conclusions are similar. The eigen-analysis for the BS antenna spacing changes revealed that the maximum eigenvalues, $\alpha_{1, q}$, do not suffer any major variation with the element spacing increase. In the remaining eigenvalues $\left(\alpha_{2, q}, \alpha_{3, q}\right)$, it exists an increase with element separation, specially from $0.5 \lambda$ to $5 \lambda$ BS antenna spacing configurations. For antenna separations higher that $5 \lambda$, the secondary eigenvalues still increase with antenna spacing, but at a lower rate.

The results are displayed in Fig. 12. The MIMO gain with uniform power allocation shows an increase with the BS antenna spacing. The MIMO gain then saturates for BS antenna spacings higher than $5 \lambda$ for both MIMO configurations.

In the water-fill case, the MIMO gain is approximately constant. The power transference to the strongest and constant eigenmodes $\left(\alpha_{1, q}\right)$, removes the moderate capacity increase effect due to the secondary eigenvalues growth with the BS antenna spacing. As a final remark, the results suggest that, for the macro-cell case, if no channel information is available, BS antenna spacing should be set between $\lambda$ and $5 \lambda$, since no additional gain increase was detected using uniform power allocation. Additionally, if channel information is provided and spatial water-filling is used, the BS antenna spacing can be even lesser, with no apparent degradation on offered channel capacity. The choice will depend on the radio environment.

\subsection{MS Antenna Spacing}

For the MS antenna element spacing impact, the BS antenna spacing is kept at $5 \lambda$.

The 3GPP [8] suggests a typical MS antenna spacing of $0.5 \lambda$. The intention is to get capacity results with similar antenna spacings in a macro-cell urban environment, in order to 


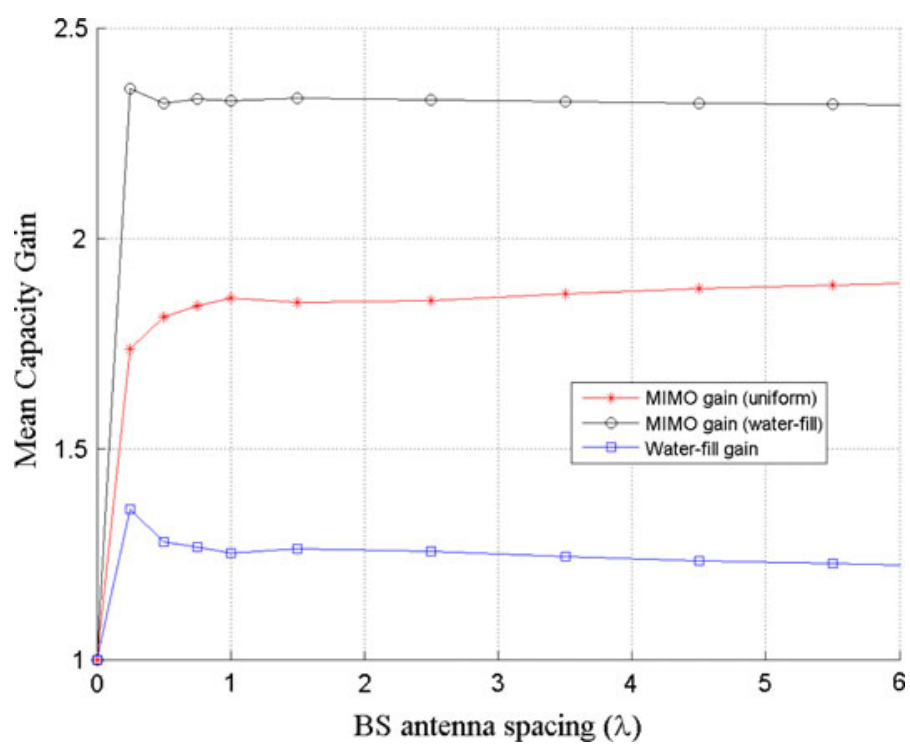

Fig. 12 MIMO and water-fill gain for MIMO $3(m \lambda) \times 3(0.5 \lambda)$ setup, SNR $=5 \mathrm{~dB}$, BS antenna spacing variation

check if this value is adequate. Having in mind the MS size limitations, the antenna separation should not exceed, say, one wavelength, $\lambda$.

The maximum separation depends naturally on the number of antenna elements, in order to keep the MS antenna array under a realistic size.

The following results are for a MIMO $2 \times 2$ setup. The eigen-analysis for the MS antenna spacing changes shows that the maximum eigenvalues $\alpha_{1, q}$ do not suffer any major variation with the element spacing increase. In the secondary eigenvalues $\left(\alpha_{2, q}\right)$, it exists an increase with element separation, specially from $0.1 \lambda$ to $0.3 \lambda$ MS antenna spacing configurations. For MS antenna separations higher that $0.3 \lambda$, the secondary eigenvalues still increases with antenna spacing, but much more slowly. As in the BS antenna spacing analysis, the MS antenna spacing mean MIMO and water-filling gains are presented in Fig. 13.

Looking at the values for the MIMO gain with uniform power allocation, the data shows an increase with the MS antenna spacing. The MIMO gain stabilizes for MS antenna spacings higher than $0.4 \lambda$.

In the water-fill case, the MIMO gain also saturates for higher antenna spacings. For shorter antenna spacings (shorter than $0.3 \lambda$ ), the $\left(\alpha_{2, q}\right)$ eigenvalue is very weak, which enables an increased water-fill gain compared with uniform power distribution, since practically no waste of power is done on the secondary eigenvalues.

The capacity MIMO gains reach a 60 and $70 \%$ increase for the uniform and water-fill power allocation, respectively. The water-fill gain is aproximately $8 \%$.

The simulated data agrees with the 3 GPP suggested $0.5 \lambda$ value [8]. With or without smart power allocation techniques and for these SNR conditions, there is no additional advantage on using MS antenna spacings larger than half of the wavelength, $0.5 \lambda$.

Considering the MS terminal size limitations, the simulations show that is preferable to maintain small element spacings of $0.5 \lambda$ and if possible add more elements, than maintain the number of elements and increase antenna spacing. The intense angular multi-path dispersion 


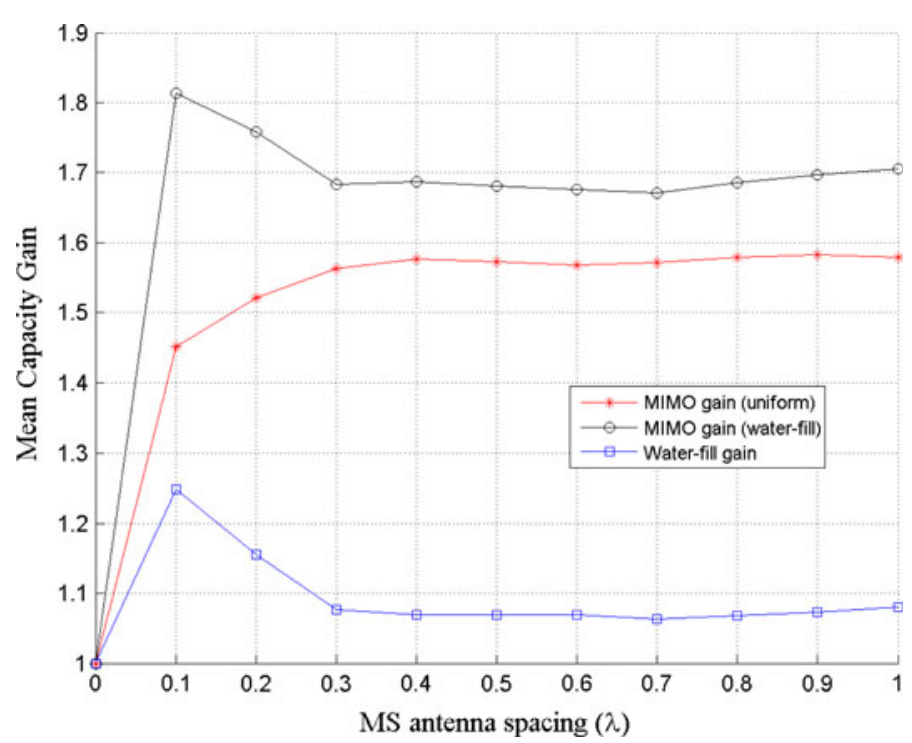

Fig. 13 MIMO and water-fill gain for MIMO $2(5 \lambda) \times 2(m \lambda)$ setup, SNR $=5 \mathrm{~dB}$, MS antenna spacing variation

in the MS surroundings enables the $0.5 \lambda$ value or even slightly lesser antenna spacing. On the other hand, smaller antenna spacings (less than $0.3 \lambda$ ) should not be implemented at the expense of considerable capacity reduction.

\subsection{Miscelaneous MIMO Configurations}

After analyzing the number of antennas and BS/MS antenna spacing impact on capacity, it is important to compare miscellaneous MIMO configurations, i.e., configurations with different number of antenna elements and antenna spacings, in order to evaluate the capacity gains and the optimized MIMO configurations for the proposed macro-cell scenario.

Section 6.1 sets that capacity increases with the number of antennas, for a fixed antenna spacing. Additionally, Sect. 6.2 concludes that no additional gain was detected on using BS antenna spacings higher than $5 \lambda$, with $\lambda$ being the wavelength. Similarly, in Sect. 6.3 and

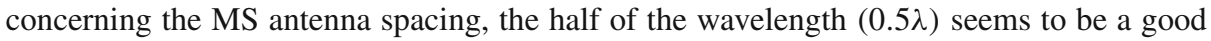
compromise solution even though other antenna spacing solutions can be appealing also. Having these considerations in mind, the BS antenna spacing will be fixed to $5 \lambda$. The total number of BS antennas will directly affect the BS array size, which is not a real constraint. The MS terminal size restrictions are much more problematic.

The number of antennas and spacing at the MS will depend on the maximum antenna array size and carrier frequency. Considering linear arrays and trying to limit the test configurations to a short and workable number, only MS antenna array configurations with a total size less or equal than $30 \mathrm{~cm}$ will be set, as already mentioned. Working at $2 \mathrm{GHz}$, the considered configurations are presented in Table 1 along with size and the capacity mean values. The respective mean eigenvalues, $\bar{\alpha}_{k, q}$ are presented in Table 2 . It consists helpful information on the analysis.

With the objective of stimulating the direct comparison between configurations, Fig. 14 was produced, displaying the MIMO and water-fill gain graphical results. The reference is 
Table 1 Mean capacity comparison for miscellaneous MIMO setups, SNR $=5 \mathrm{~dB}$

\begin{tabular}{llll}
\hline Configuration & MS size $(\mathrm{cm})$ & Capacity (uniform) $(\mathrm{bit} / \mathrm{s} / \mathrm{Hz})$ & Capacity (water-fill) $(\mathrm{bit} / \mathrm{s} / \mathrm{Hz})$ \\
\hline SISO & - & 2.06 & 2.06 \\
$2(5 \lambda) \times 2(0.5 \lambda)$ & 7.5 & 3.24 & 3.46 \\
$2(5 \lambda) \times 2(1 \lambda)$ & 15.0 & 3.25 & 3.51 \\
$2(5 \lambda) \times 2(1.5 \lambda)$ & 22.5 & 3.26 & 3.55 \\
$3(5 \lambda) \times 3(0.5 \lambda)$ & 15.0 & 4.34 & 5.02 \\
$3(5 \lambda) \times 3(1 \lambda)$ & 30.0 & 4.37 & 5.06 \\
$4(5 \lambda) \times 4(0.5 \lambda)$ & 22.5 & 4.63 & 5.85 \\
$5(5 \lambda) \times 5(0.5 \lambda)$ & 30.0 & 4.71 & 6.23 \\
\hline
\end{tabular}

Table 2 Mean eigenvalues, $\bar{\alpha}_{k, q}$, for miscellaneous MIMO setups

\begin{tabular}{lccccc}
\hline Configuration & $\bar{\alpha}_{1, \mathrm{q}}(\mathrm{dB})$ & $\bar{\alpha}_{2, \mathrm{q}}(\mathrm{dB})$ & $\bar{\alpha}_{3, \mathrm{q}}(\mathrm{dB})$ & $\bar{\alpha}_{4, \mathrm{q}}(\mathrm{dB})$ & $\bar{\alpha}_{5, \mathrm{q}}(\mathrm{dB})$ \\
\hline SISO & 0 & - & - & - & - \\
$2(5 \lambda) \times 2(0.5 \lambda)$ & 5.9 & -9.0 & - & - & - \\
$2(5 \lambda) \times 2(1 \lambda)$ & 5.9 & -8.4 & - & - & - \\
$2(5 \lambda) \times 2(1.5 \lambda)$ & 5.9 & -8.3 & - & - & - \\
$3(5 \lambda) \times 3(0.5 \lambda)$ & 9.2 & -2.7 & -13.5 & - & - \\
$3(5 \lambda) \times 3(1 \lambda)$ & 9.2 & -2.3 & -12.7 & - & - \\
$4(5 \lambda) \times 4(0.5 \lambda)$ & 11.5 & 2.1 & -5.6 & -16.4 & - \\
$5(5 \lambda) \times 5(0.5 \lambda)$ & 13.5 & 3.2 & -3.0 & -10.0 & -20.9 \\
\hline
\end{tabular}

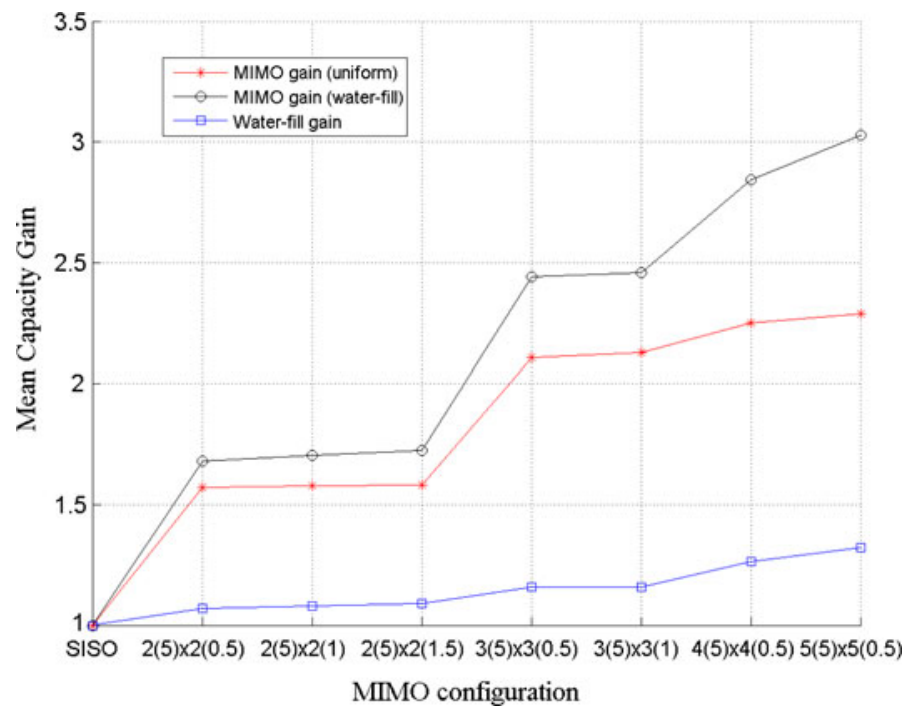

Fig. 14 MIMO and water-fill gain for miscellaneous MIMO setups, SNR $=5 \mathrm{~dB}$ 
the SISO. The graph's MIMO labels are, using previously defined terminology, $R\left(d_{r} / \lambda\right) \times$ $S\left(d_{s} / \lambda\right)$.

Some conclusions can be extracted from the presented results. MIMO $5(5 \lambda) \times 5(0.5 \lambda)$ configuration is the one that achieves higher spectral efficiency values with 6.2 and 4.7 $\mathrm{bit} / \mathrm{s} / \mathrm{Hz}$ with water-filling and uniform power allocations, respectively, which represents a remarkable growth of 300 and $230 \%$ when compared with the SISO configuration (reference). The larger spectral efficiency in the MIMO setups with additional antennas is obviously due to the contribution of the stronger $\alpha_{1, q}$ eigenvalues and also due to the modest contribution of the remaining ones.

The balancing between adding more antennas and increasing the antenna spacing can be linked with the MS antenna size. If we compare identical MS antenna size configurations like MIMO $2(5 \lambda) \times 2(1 \lambda)$ and $3(5 \lambda) \times 3(0.5 \lambda)$ or even $3(5 \lambda) \times 3(1 \lambda)$ and $5(5 \lambda) \times 5(0.5 \lambda)$, one can conclude that it is always the one with more antennas and less antenna spacing that achieves the highest capacity. This is explained by the additional sub-channel creation which will increase capacity. So, adding more antennas is more powerful in terms of capacity increase, than increasing antenna separation, when comparing equal size configurations.

Considering a capacity versus MS antenna length ratio (in bit $/ \mathrm{s} / \mathrm{Hz} / \mathrm{cm}$ ), the top three configurations in descendant order are the $2(5 \lambda) \times 2(0.5 \lambda), 3(5 \lambda) \times 3(0.5 \lambda)$ and $4(5 \lambda) \times 4(0.5 \lambda)$ which reinforces the antenna addition vs. antenna array separation.

Water-filling scheme provides equal or higher capacity compared with the uniform power scheme, as expected. The capacity increase difference between uniform power allocation and water-filling usage is greater in MIMO configurations with higher number of antenna elements, due to the stronger and increased number of eigen-modes.

\section{MIMO SNR Impact}

Section 6 analyzed the MIMO configuration impact using previously chosen configuration setups for a $5 \mathrm{~dB}$ SNR. It is equally important to study if the conclusions and recommendations issued for that specific SNR are valid for lower or even higher values.

New simulations were computed for different SNR values. The same MIMO channel model setup was used (see Sect. 5), for the eigen and capacity analysis. The final capacity CDFs (using uniform and spatial water-filling) are presented in Figs. 15 and 16 assuming the MIMO $2(5 \lambda) \times 2(0.5 \lambda)$ setup. The implemented SNRs vary between $0 \mathrm{~dB}$ and $30 \mathrm{~dB}$ with a $5 \mathrm{~dB}$ interval. As expected, the capacity increases with SNR and the spatial water-filling guarantees always better capacity than the uniform power distribution.

In order to evaluate the capacity increase as a function of SNR, mean capacities using the two power distributions were extracted, and displayed in Figs. 17 and 18 for different MIMO configurations. The MIMO setups are the same as in Sect. 6.4 and named as miscellaneous MIMO configurations, i.e., configurations with different number of antenna elements and antenna spacings. Once again the objective is to achieve optimized MIMO configurations for the proposed macro-cell scenario, counting now with the information about SNR variation.

Analysing the data, one can conclude that the capacity increases with the number of antennas and SNR. The first dependence was already noticed in Sect. 6.1 but reinforced by the last simulations.

At lower SNR, the contribution of the strongest spatial sub-channels are dominant $\left(\alpha_{1, q}\right)$. For higher SNRs all the additional sub-channels contribute to the total capacity, which corroborates the curves's slope increase with SNR. The MIMO $5(5 \lambda) \times 5(0.5 \lambda)$ is the best one 


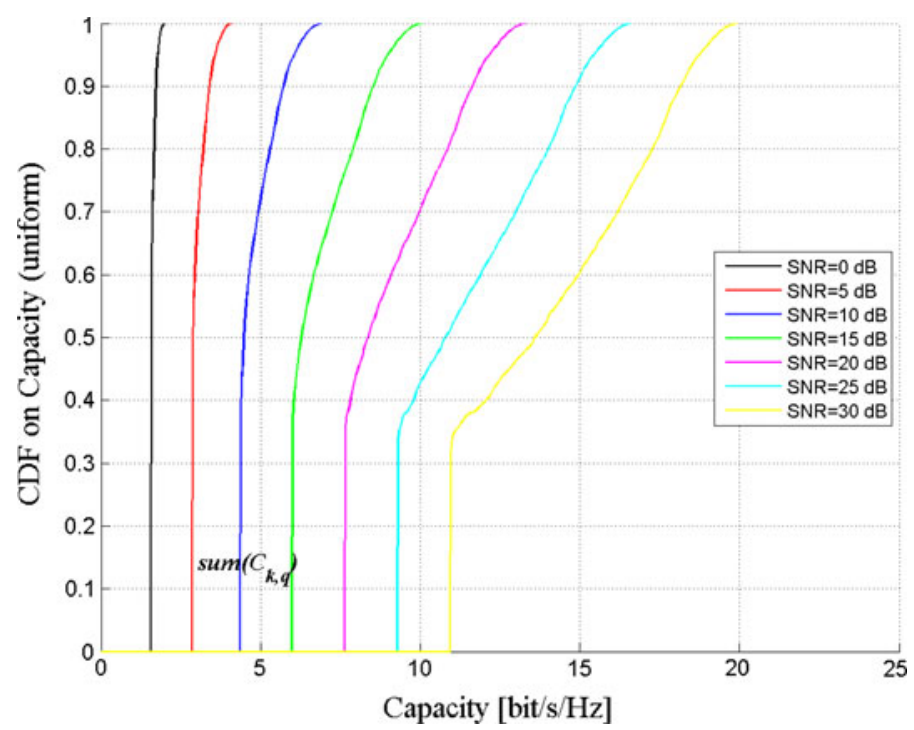

Fig. $15 \mathrm{CDF}$ of the total capacity using uniform power distribution algorithm. MIMO $2(5 \lambda) \times 2(0.5 \lambda)$ setup; antenna spacing; $\mathrm{SNR}=0,5,10,15,20,25,30 \mathrm{~dB}$

Fig. $16 \mathrm{CDF}$ of the total capacity using spatial water-filling power distribution algorithm. MIMO $2(5 \lambda) \times 2(0.5 \lambda)$ setup; antenna spacing; SNR $=0,5,10,15,20$, $25,30 \mathrm{~dB}$

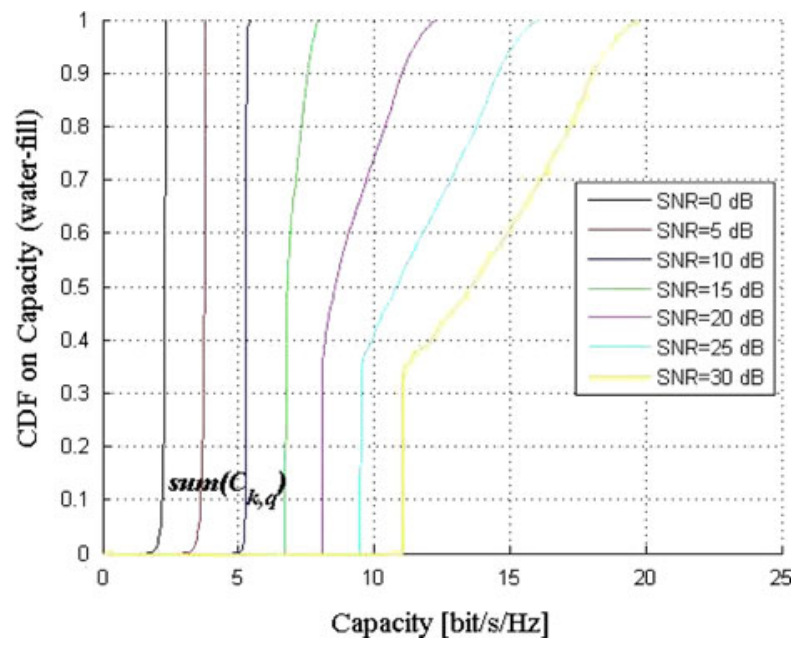

in terms of performance and takes full advantage of its additional sub-channels compared with the other configurations.

It was already noticed that, for a fixed SNR, the capacity boost that is achieved when passing from SISO to a two element MIMO setup is considerably higher than passing from two to three element configuration, and so on. Even if always higher capacity is reached, increasing the number of elements in MIMO setups will not produce proportional capacity augmentation. Considering also the MIMO configurations complexity increase in design and signal processing level, many antenna MIMO usage gets even harder.

Comparing identical configurations in what concerns the number of antennas, the MS antenna spacing variation can be highlighted as well as its impact on capacity for different 


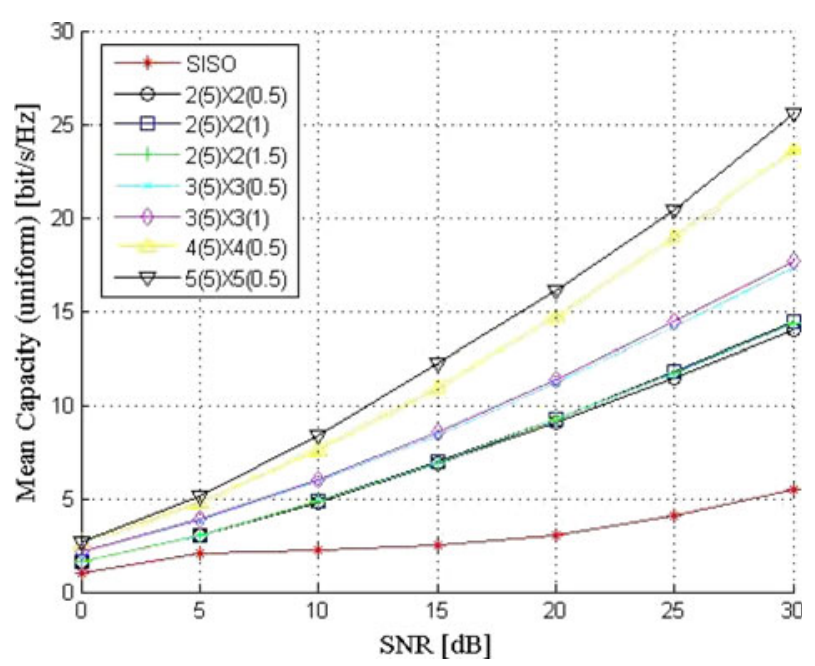

Fig. 17 Mean Capacity versus SNR for miscelaneous MIMO configurations using uniform power allocation

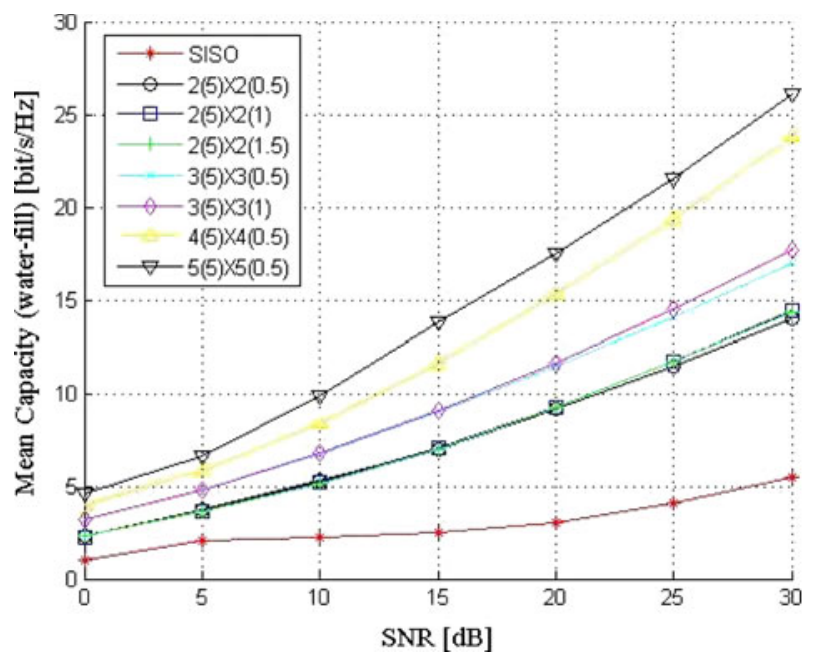

Fig. 18 Mean Capacity versus SNR for miscelaneous MIMO configurations using spatial water-filling

SNRs. As in Sect. 6.3, the MS antenna spacing factor produces a moderate increase in channel capacity, which grows with the SNR. Analysing Table 2, it is noticed that increasing antenna element spacing, $\alpha_{1, q}$ does not suffer any major changes. Indeed, it was the other eigenvalues that experienced an increase. At low SNR, the contribution of the strongest spatial sub-channel is dominant but, for higher SNR, the remaining sub-channels also give considerable contribute to the total capacity. As a consequence, secondary spatial sub-channels already reinforced due to antenna spacing increase, have a stronger contribute to the total capacity as the SNR increases. This explains the higher distinction between curves for equal number of antenna as the SNR improves. 


\section{Conclusions}

In this paper, a new FS MIMO framework for macro-cells in a realistic urban environment has been developed. The MIMO model is built over a previously developed directional channel model, which is an extension of COST 273 channel model, introducing terrain and building information into radio channel simulation.

The effect of spatial water-filling on the capacity of FS MIMO systems was analyzed. It has been shown that the spatial water-filling results in an increased channel capacity compared with uniform transmit power allocation. The analysis was based on the selective channel matrix eigenvalue distribution and quantified by the channel capacity measure.

Recall that the presented space/frequency eigenvalues and capacity results are theoretical values for error free transmission and provide an idea of the MIMO performance. To achieve these values in real world, coding, constellation size among others should be considered in the system implementation. For an isolated MIMO system, the results presented here are a theoretical upper limit to the associated data rate, but can be used for comparison purposes.

Next, MIMO configuration characteristics were investigated in order to maximize capacity, mainly number of antennas, inter-element spacing and SNR. Channel and capacity simulation results were presented for the city of Lisbon, Portugal. Two power allocations schemes were considered: uniform distribution and FS spatial water-filling. The results suggested optimized MIMO configurations, considering the antenna array size limitations, mostly on the MS side. The main ideas are summarized in the following.

The MIMO capacity increases with the number of antennas and SNR, as expected.

If no channel information is available, BS antenna spacing should be set between $\lambda$ and $5 \lambda$, since no additional gain increase was detected using uniform power allocation. Additionally, if channel information is provided and spatial water-filling is used, the BS antenna spacing can be even lesser, with no apparent degradation on offered channel capacity. The choice will depend on the radio environment.

The simulations agree with the 3GPP suggested $0.5 \lambda$ value [8]. With or without smart power allocation techniques and for the simulated SNR conditions, there is no additional advantage on using MS antenna spacings larger than half of the wavelength $0.5 \lambda$, whatever the SNR.

Considering a capacity versus MS antenna length ratio (in bit $/ \mathrm{s} / \mathrm{Hz} / \mathrm{cm}$ ), the top three configurations in descendant order are the $2(5 \lambda) \times 2(0.5 \lambda), 3(5 \lambda) \times 3(0.5 \lambda)$ and $4(5 \lambda) \times$ $4(0.5 \lambda)$. eigenvalues.

Water-filling scheme provides equal or higher capacity compared with the uniform power scheme, as expected. The capacity increase between uniform power allocation and waterfilling usage is greater in MIMO configurations with higher number of antennas, due to the stronger and increased number of eigen-modes.

\section{References}

1. Foschini, G. J., \& Gans, M. J. (1998). On limits of wireless communications in a fading environment when using multiple antennas. Wireless Personal Communications, 6, 311-335.

2. Telatar, E. (1995). Capacity of multiantenna gaussian channels. AT\&T Bell Laboratories, Technical Memorandum.

3. Cover, T. M., \& Thomas, J. A. (1991). Elements of information theory. New York: Wiley.

4. Vieira, P., \& Queluz, M. P., Rodrigues, A. (2007). Terrain and clutter impact on joint statistical properties of azimuth spread and delay spread in macro-cell bad urban environment. In Proceedings of 
IEEE international symposium on wireless communication systems 2007 (pp. 798-802). Trondheim, Norway.

5. Vieira, P., Queluz, M. P., \& Rodrigues, A. (2007). Clustering of scatterers over an irregular clutter environment: An extension of cost 273 mimo channel model. In Proceedings of 66th IEEE vehicular technology conference fall 2007 (pp. 824-828). Baltimore, USA.

6. Vieira, P., Queluz. M. P., \& Rodrigues, A. (2007). A dynamic propagation prediction platform over irregular terrain and buildings for wireless communications. In Proceedings of 66th IEEE vehicular technology conference fall 2007 (pp. 884-888). Baltimore, USA.

7. Vieira, P., Vieira, M. A., Queluz, M. P., \& Rodrigues, A. (2007). A novel vehicular mobility model for wireless networks. Wireless Personal Communications Journal, Springer, 43, 1689-1703.

8. 3GPP. (2007). Spatial channel model for multiple input multiple output (mimo) simulations. 3GPP TR 25.996 V7.0.0.

9. Andreas, F. M. (2005). Wireless communications. New York: Wiley.

10. Jakes, W. C. (1993). Microwave mobile communications. Piscataway, NJ: IEEE Press.

11. Vieira, P., Queluz, M. P., \& Rodrigues, A. (2007). An improved directional channel model over irregular terrain and clutter. In Proceedings of 10th international symposium of wireless personal multimedia communications 2007 (pp. 523-527). Jaipur, India.

12. Andersen, J. B. (2000). Array gain and capacity for known random channels with multiple element arrays at both ends. IEEE JSAC, 18(11), 2172-2178.

13. Kermoal, J. P., Schumacher, L., Pederson, K. I., \& Mogensen, P. E. (2002). A stochastic mimo radio channel model with experimental validation. IEEE JSAC, Special Issue on Channel and Propagation Models for Wireless System Design, 20(6), 1211-1226.

14. Almers, P., Tufvesson, F., Edfors, O., \& Molisch, A. F. (2002). Measured capacity gain using water filling in frequency selective mimo channels. In The 13th IEEE international symposium on personal, indoor and mobile radio communications, 2002, 3, 1347-1351.

\section{Author Biographies}

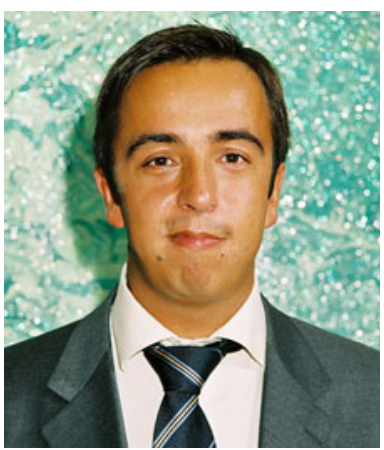

Pedro Vieira was born in Lisbon, Portugal, in 1974. He received the Eng., the M.Sc. and the Ph.D. degree in Electrical and Computer Engineering from Instituto Superior Técnico (IST), Technical University of Lisbon, Portugal, in 1997, 2003 and 2008, respectively. He is currently an Assistant Professor in Lisbon Polytechnic Institute (ISEL) and a Researcher at Instituto de Telecomunicações, where he is researching aspects of wireless communications and MIMO systems.

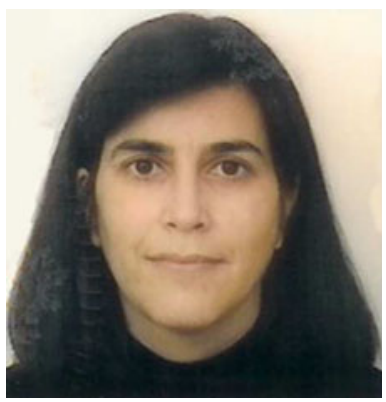

Paula Queluz received the E.E. and the M.Sc. degrees in electrical and computer engineering from the Instituto Superior Técnico, Technical University of Lisbon, Portugal, in 1985 and 1989, respectively, and the Ph.D. degree in Applied Sciences from the Catholic University of Leuven, Belgium, in 1996. She is presently Assistant Professor at the Technical University of Lisbon and researcher at Instituto de Telecomunicações, Lisbon. Her scientific interests include image analysis/processing, copyright protection and mobile communications. 


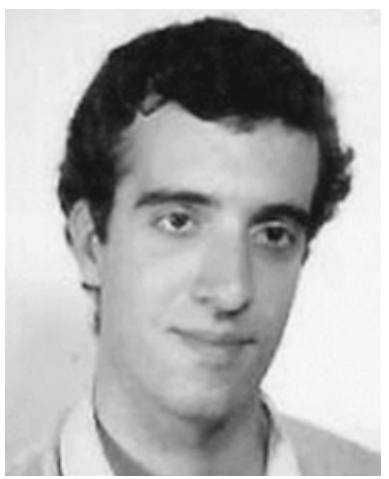

António Rodrigues received the B.S. and M.S degrees in electrical and computer engineering from the Instituto Superior Técnico (IST), Technical University of Lisbon, Lisbon, Portugal, in 1985 and 1989, respectively, and the Ph.D. degree from the Catholic University of Louvain, Louvain-la-Neuve, Belgium, in 1997. Since 1985, he has been with the Department of Electrical and Computer Engineering, IST, where is currently an Assistant Professor. His current interests include modulation, coding, multiple access techniques, cellular mobile radio systems, digital radio transmission and diversity reception and equalization problems for mobile environments including satellite/mobile systems. 